dex versehen. So können Ärzte unkompliziert in der Cloud nach Patientendaten suchen, wenn sie zum Beispiel herausfinden wollen, welche Patienten wieder für einen Check-up oder eine andere Präventionsleistung angesprochen werden sollten. Es müssen nicht erst alle verschlüsselten Datensätze auf den Praxisrechner herunterladen werden, um die Daten wieder komplett zu entschlüsseln und eine Klartextsuche starten zu können. Spalka: „Wenn Sie einen Datensatz zurückholen müssen, ist das o.k., wenn Sie aber 20000 Datensätze erst herunterladen und dann entschlüsseln müssen, sieht es schon anders aus."

\section{Datensuche in Sekundenschnelle}

Dass die Suche in den verschlüsselten Daten funktioniert, zeigte das Unternehmen vergangenen November auf der Medica in Düsseldorf. Wer nach den Daten eines speziellen Patienten sucht, gibt dessen Namen ein und hat in wenigen Sekunden alle Infos parat. Fast so, als lägen die Daten direkt auf dem Praxisrechner vor Ort. Das System bietet zusätzlich eine phonetische Suche, falls die
MFA am Empfang den Patientennamen einmal nicht richtig verstanden hat. Praxen können aber auch ihre gewohnten Schnellstatistiken etwa zu den Patienten, die in den nächsten Tagen Geburtstag haben oder - wie bereits erwähnt die das Team demnächst wieder auf eine Präventionsleistung ansprechen könnte, weiter nutzen. Auch hier liefert die Suche in der Cloud zügig die Daten. Dabei erhält jede Praxis in der Cloud ihre eigene Datenbank, erklärte Dr. Tobias Rho, Sicherheitsexperte bei CGM.

\section{Eine Super-PIN zur Verschlüsselung}

Beim ersten Prototypen, den der HealthIT-Anbieter in Düsseldorf vorstellte, legt sich die Praxis ihren Schlüssel für den Zugriff auf die Cloud und die Verschlüsselung der Daten selbst an. Während dieses Prozesses wird für die Praxis eine sogenannte Super-PIN generiert. Mit dieser kann die Praxis, ähnlich wie bei Handyverträgen, einen neuen Zugriffsschlüssel anfertigen, falls der Arzt einmal seinen Schlüssel vergisst oder durch mehrfache Falscheingabe blockiert.
CGM kann den Schlüssel nicht zurücksetzen. Damit soll sichergestellt werden, dass tatsächlich nur der Arzt die Daten entschlüsseln kann. Die Super-PIN sollte sich die Praxis daher gut aufbewahren. Ist diese weg, kommt nämlich keiner mehr an die Daten.

Was die Medical Cloud ebenfalls möglich macht: Es können verschiedene Schlüsselarten für verschiedene $\mathrm{Zu}$ griffsrechte vergeben werden. So erhält eine Praxismitarbeiterin, die nur für die Abrechnung beschäftigt wird, beispielsweise nur einen Schlüssel für den Zugriff auf Abrechnungsdaten. Und: Es lassen sich verschiedene Schlüssel für unterschiedliche Praxiseinheiten anlegen. Das ist laut CGM etwa für MVZ interessant, denn dort könnten sich einzelne Ärzte in der Cloud eine eigene Dateneinheit anlegen.

Der Zugriff auf die Medical Cloud wird aber nicht nur über feststehende Rechner oder Notebooks möglich sein. Auch mobile Geräte wie Tablet-PC können mit der Cloud kommunizieren. Allerdings nutzt CGM erst einmal nur Android-Systeme.

Rebekka Höhl

\title{
Kassen nutzen die Chancen der IT zu wenig
}

Das deutsche Gesundheitssystem hinkt informationstechnisch anderen Branchen hinterher.

Hier liegen erhebliche Effizienzreserven.

$\mathrm{M}$ edizinisch-technisch sei die Gesundheitsversorgung in Deutschland gut aufgestellt, in der Organisation und in der administrativ-medizinischen Ausstattung seien jedoch Optimierungen nötig, um immanente Schwächen wie die Trennung von ambulantem und stationärem Sektor zu überwinden und die Produktivität zu erhöhen, urteilt das Beratungsunternehmen Lünendonk in seinem Branchendossier „Healthcare 2020“. Steigende Ausgaben, sinkende Einnahmen sowie ineffiziente Strukturen ließen das System mittelfristig havarieren.

Das Rheinisch-Westfälische Institut für Wirtschaftsforschung e.V. schätze die Finanzlücke auf über 90 Milliarden $€$ bis 2030. Dies bedeute, dass der GKVBeitrag von derzeit $15,5 \%$ auf $23,8 \%$ oder der Zusatzbeitrag auf $115 €$ steigen müsse. Das werde die Bevölkerung nicht zahlen können und wollen. Die politische Reformfähigkeit des Systems sei begrenzt, dirigistische Eingriffe in Angebot und Nachfrage zeigten zwar Wirkung, reichten aber nicht aus, so Lünendonk. Bessere Leistungssteuerung und eine Erhöhung der Effizienz seien daher zusätzlich erforderlich. Informationsund Kommunikationstechnik wäre ein wirksames Mittel, wie das Beispiel aller anderen Wirtschaftsbereiche zeige. Erreichen ließe sich mehr Produktivität in der Gesundheitsversorgung laut Dossier durch
— Vermeidung von Leistungskosten mittels Prävention und Leistungssteuerung,

_organisatorische Integration unter Kosten-Nutzen-Aspekten

_ und Steigerung der Effizienz der integrierten Versorgung mittels technischer und datentechnischer Innovationen. Wie diese aussehen könnte, wird in dem Dossier anhand eines fiktiven Herz-OP-Patienten dargestellt, der Zuhause telemedizinisch betreut wird.

Monika Peichl
Branchendossier "Healthcare 2020"

Das knapp 100-seitige Dossier kann unter http://bit.ly/Y0ko1C kostenlos heruntergeladen werden. 